\title{
Shifts in Borrelia burgdorferi (s.l.) geno- species infections in Ixodes ricinus over a 10-year surveillance period in the city of Hanover (Germany) and Borrelia miyamotoi- specific Reverse Line Blot detection
}

\author{
Katrin Blazejak', Marie-Kristin Raulf1,2, Elisabeth Janecek ${ }^{1}$, Daniela Jordan ${ }^{1}$, Volker Fingerle ${ }^{3}$ and Christina Strube ${ }^{1 *}$
}

\begin{abstract}
Background: Lyme borreliosis caused by spirochetes of the Borrelia burgdorferi (sensu lato) complex is still the most common tick-borne disease in Europe, posing a considerable threat to public health. The predominant vector in Europe is the widespread hard tick Ixodes ricinus, which also transmits the relapsing fever spirochete $B$. miyamotoi as well as pathogenic Rickettsiales (Anaplasma phagocytophilum, Rickettsia spp.). To assess the public health risk, a long-term monitoring of tick infection rates with the named pathogens is indispensable.

Methods: The present study is the first German 10-year follow-up monitoring of tick infections with Borrelia spp. and co-infections with Rickettsiales. Furthermore, a specific Reverse Line Blot (RLB) protocol for detection of $B$. miyamotoi and simultaneous differentiation of B. burgdorferi (s.l.) geno-species was established.

Results: Overall, 24.0\% (505/2100) of ticks collected in the city of Hanover were infected with Borrelia. In detail, 35. $4 \%(203 / 573)$ of adult ticks [38.5\% females (111/288) and 32.3\% males (92/285)] and 19.8\% nymphs (302/1527) were infected, representing consistent infection rates over the 10-year monitoring period. Geno-species differentiation using RLB determined B. miyamotoi in 8.9\% (45/505) of positive ticks. Furthermore, a significant decrease in B. afzelii and B. spielmanii infection rates from 2010 to 2015 was observed. Co-infections with Rickettsia spp. and $A$. phagocytophilum increased between 2010 and 2015 (7.3 vs 10.9\% and 0.3 vs 1.1\%, respectively).

Conclusions: Long-term monitoring is an essential part of public health risk assessment to capture data on pathogen occurrence over time. Such data will reveal shifts in pathogen geno-species distribution and help to answer the question whether or not climate change influences tick-borne pathogens.
\end{abstract}

Keywords: Lyme disease, Tick-borne diseases, Ticks, Borrelia, Rickettsiales, Borrelia miyamotoi

\section{Background}

Lyme borreliosis (LB) is still the most common tickborne disease in the Northern Hemisphere and poses a considerable risk to public health [1]. Estimated LB incidences in Europe total approximately 85,000 cases annually [2]. Data from mandatory notifications from six federal states in the eastern part of Germany show that

\footnotetext{
* Correspondence: christina.strube@tiho-hannover.de

${ }^{1}$ Institute for Parasitology, Centre for Infection Medicine, University of Veterinary Medicine Hannover, Buenteweg 17, 30559 Hanover, Germany Full list of author information is available at the end of the article
}

the overall incidence varied between 34.9 cases/100,000 inhabitants in 2009 and 19.54 cases/100,000 inhabitants in 2012 [3]; extrapolation to the total German population results in approximately 15,000 to 30,000 LB cases per year. The resulting impact on public health care systems is substantial due to annual medical costs, which are estimated for Germany alone to be 23.7 million Euros for LB hospital care and 51.2 million Euros for outpatients. Additionally, indirect costs due to a loss of productivity are estimated at 7.1 million Euros annually [4]. In Europe, B. burgdorferi (sensu lato) spirochetes are

(c) The Author(s). 2018 Open Access This article is distributed under the terms of the Creative Commons Attribution 4.0 International License (http://creativecommons.org/licenses/by/4.0/), which permits unrestricted use, distribution, and reproduction in any medium, provided you give appropriate credit to the original author(s) and the source, provide a link to the Creative Commons license, and indicate if changes were made. The Creative Commons Public Domain Dedication waiver (http://creativecommons.org/publicdomain/zero/1.0/) applies to the data made available in this article, unless otherwise stated. 
mostly transmitted by their main vector, the castor bean tick Ixodes ricinus [1]. To date, 22 geno-species of the $B$. burgdorferi (s.l.) complex have been identified, with 11 of them occurring throughout Europe [5].

In addition, $B$. miyamotoi, belonging to the relapsing fever (RF) borreliae, has been detected in I. ricinus in Europe, which also represents their main vector as opposed to related RF borreliae [6-8]. Borrelia burgdorferi (s.l.) prevalences in ticks show large differences nationwide [9-18] with exemplarily 3.1\% Borrelia-positive ticks at the German Baltic coast [13] and considerably higher infection rates of $34.1 \%$ in the northern German city of Hamburg [12] or $36.2 \%$ in the southern federal state Bavaria [14]. Conversely, B. miyamotoi tick infection rates vary between $1.8 \%$ [18] and $2.4 \%$ in western as well as $2.7 \%$ in southern Germany [19], whereas data from northern Germany are not yet available. In humans, $B$. miyamotoi mostly causes influenza-like illnesses with fever, nausea, fatigue, chills, headache or myalgia [6, 20], resulting in challenging diagnostics as symptoms are rather unspecific. However, severe cases of B. miyamotoiinduced meningoencephalitis have been described in immunocompromised patients [21, 22].

Here, we present long-term data in terms of a 10year follow-up monitoring on tick infection rates with Borrelia spp. and co-infections with Rickettsiales in the city of Hanover, Germany, to contribute to public health risk assessment.

\section{Methods}

\section{Tick material and sampling sites}

From April to October 2015, a total of 2100 questing ticks were collected by the flagging method at ten different recreation areas evenly distributed in the city of Hanover as described previously [23]. The locations comprised extensive forest areas as well as inner urban parks, frequently visited by inhabitants and tourists. The study design was based on previous studies conducted in 2005 and 2010 [9-11] and served simultaneously as a 10-year follow-up monitoring. A total of 30 ticks per month was collected at the same sampling locations previously examined in 2010 and 2005 [9-11]. However, due to construction measures, two sampling sites differed between 2015 and 2010 compared to 2005 .

Tick species and developmental stage identification of collected ticks was performed according to morphological keys described by Estrada-Peña et al. [24]. Ticks were individually stored at $-20^{\circ} \mathrm{C}$ until genomic DNA isolation.

\section{Genomic DNA isolation and detection of Borrelia spp. by quantitative real-time PCR}

Ticks were individually homogenized and genomic DNA isolation was performed as described elsewhere $[23,25,26]$. Isolated DNA was stored at $-20{ }^{\circ} \mathrm{C}$ until further use. A qPCR targeting the 5S-23S rRNA intergenic spacer (IGS) based on a primer-probe combination designed by Strube et al. [11] was performed as described previously $[9,12]$.

\section{Design of a B. miyamotoi-specific Reverse Line Blot (RLB) probe}

For detection of B. miyamotoi by Reverse Line Blot (RLB), a species-specific probe was designed based on seven published sequences targeting the hydrolase-23S rRNA IGS of B. miyamotoi. Sequences were retrieved from the GenBank database (National Centre for Biotechnology Information) under accession numbers GQ387038 (Switzerland), CP017126, AY531879, CP010308, CP006647 (USA) and CP004217 (Japan). Furthermore, B. miyamotoi (strain HT 31) DNA was amplified by RLB-PCR using primers B-5SBor and 23SBor [27] to obtain a $400 \mathrm{bp}$ fragment of the hydrolase-23S rRNA IGS. The PCR product was cloned and sequenced to identify candidate regions for probe design. Therefore, the obtained sequence of $B$. miyamotoi strain HT 31 was aligned with named sequences (GenBank: GQ387038, CP017126, AY531879, CP010308, CP006647 and CP004217) using the Clone Manager Professional software (version 9, Scientific and Educational Software, Denver, USA).

\section{Reverse Line Blot (RLB)}

To determine geno-species of Borrelia spp. positive tick samples, the RLB technique was performed as described previously $[9,12]$ with few modifications. RLB-PCR included biotin-linked B5S-Bor forward and 23S-Bor reverse primer as described by Alekseev et al. [27] to amplify the B. burgdorferi (s.l.) 5S-23S rRNA IGS target region, which occurs as duplicate in the genome of the B. burgdorferi (s.l.) complex. For amplification of $B$. miyamotoi, a hydrolase-23S rRNA region specific, biotin-linked forward primer was designed and included in the reaction mixture (BMiya-For: 5'-TTA GGA TTA ATG ATR TTK TTA CC-3'). The $25 \mu$ reaction mixture contained $1 \mu \mathrm{l}$ B5S-Bor forward primer and $1 \mu$ BMiyaFor in addition to $2 \mu \mathrm{l} 23 \mathrm{~S}$-Bor reverse primer $(10 \mu \mathrm{M}$ each), $0.5 \mu \mathrm{l}$ deoxynucleoside triphosphates $(10 \mathrm{mM}$

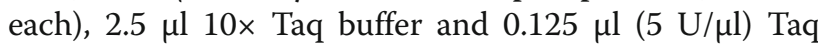
DNA polymerase (Qiagen, Hilden, Germany). Ten microlitres of tick DNA sample or $1 \mu \mathrm{l}(0.1 \mathrm{ng} / \mu \mathrm{l})$ control Borrelia DNA was utilized and the amount of double-distilled water adjusted accordingly. Controls comprised 14 Borrelia strains, which were included in each run: B. afzelii (strain PBas), B. garinii (PWudII), $B$. bavariensis (Pbi), B. bissettiae (DN127), B. burgdorferi (s. s.) (PAbe), B. lusitaniae (PotiB2), B. spielmanii (PHap), B. valaisiana (VS116), B. carolinensis, B. kurtenbachii (25015), B. miyamotoi (HT31), B. hermsii (DSM4682), B. 
recurrentis and B. duttonii. Evaluation of MIYA probe additionally included Treponema phagedenis and Leptospira spp. DNA to exclude cross-reaction with related spirochetes. PCR cycling conditions included an initial activation step at $94{ }^{\circ} \mathrm{C}$ for $3 \mathrm{~min}$, followed by 45 cycles of $94{ }^{\circ} \mathrm{C}$ for $20 \mathrm{~s}, 52{ }^{\circ} \mathrm{C}$ for $30 \mathrm{~s}, 72{ }^{\circ} \mathrm{C}$ for $30 \mathrm{~s}$ and a final elongation step at $72{ }^{\circ} \mathrm{C}$ for $7 \mathrm{~min}$. Subsequently, $20 \mu \mathrm{l}$ of tick PCR products as well as $10 \mu \mathrm{l}$ of positive controls and a no-template control were hybridized to 8 different oligonucleotide probes linked to the membrane as described previously $[9,10]$ with the following modifications of probe concentrations: B. burgdorferi (s.l.) (SL2): $0.67 \mu \mathrm{M}$ [9]; B. afzelii (AF): $0.03 \mu \mathrm{M}$ [28]; B. garinii (GA): $0.67 \mu \mathrm{M}$ [28]; B. bissettiae (BISNE2): $0.67 \mu \mathrm{M}$ [29]; B. burgdorferi (s.s.) (SS): $0.67 \mu \mathrm{M}$ [29]; B. lusitaniae (LUSINE2): $0.67 \mu \mathrm{M}$ [29]; B. spielmanii (SpiNE3): 6.7 $\mu \mathrm{M}$ [30]; B. valaisiana (VSNE): $0.67 \mu \mathrm{M}$ [30]; and $B$. miyamotoi (MIYA): $0.67 \mu \mathrm{M}$ (this study).

Chemiluminescent signals were detected using Amersham $^{\text {tw }}$ ECL $^{\mathrm{m}}$ Prime Western Blotting Detection Reagent (GE Healthcare Life Sciences, Freiburg, Germany) according to the manufacturer's instructions, followed by signal detection for a duration of $30 \mathrm{~s}$ to 3 min light exposition using Chemiluminescent-Imager Celvin ${ }^{\circ} \mathrm{S} 320+$ (Biostep, Jahnsdorf, Germany).

As the B. garinii (GA) probe detects both B. garinii and $B$. bavariensis, Sanger sequencing of positive samples was performed to differentiate between these geno-species as described previously $[9,12]$. Similarly, the B. burgdorferi (s.s.) (SS) probe detects B. burgdorferi (s.s.) and B. carolinensis. On some blots weak signals of the $B$. bissettiae (BISNE2) probe with $B$. kurtenbachii were also observed. Thus, samples showing a positive RLB signal for these probes were also sent for custom Sanger sequencing.

\section{Statistical analyses}

Data were statistically analysed by the Chi-square test with subsequent Bonferroni-Holm correction or Fisher's exact test, depending on sample sizes, as carried out previously $[9,10]$. Data analyses included comparison of infection rates between stages (total ticks, adult females, adult males, nymphs), sampling months and locations. Furthermore, comparison among stages, locations and seasons between 2015 and 2010 as well as 2005 was performed. As sampling locations, months and number of collected ticks were identical in the years 2010 and 2015 and nearly identical in 2005 (two locations differed due to construction measures), a direct comparison of obtained data between study years was feasible. For comparison of stage-related infection rates, data obtained in 2005 was included as reported by Tappe et al. [9]. To enable data comparison of the seasonal distribution over the 10-year sampling period, data obtained in 2010 and 2005 were modified according to data obtained in 2005
[31]. As the sampling periods in 2010 and 2015 started in April, instead of March/April as in 2005 [31], statistical analyses were not feasible for these sampling months. Due to variations of two sampling sites between 2005 and the follow-up studies conducted in 2010 and 2015, comparison of local distribution was not feasible for two sampling sites ("Mecklenheide" and "Seelhorster Wald") between 2005 vs 2010 and 2015. All analyses were performed with the GraphPad Prism ${ }^{\mathrm{Tm}}$ software (version 6.03, La Jolla, CA, USA).

\section{Results}

\section{Tick material}

Based on macroscopic identifiable morphologic parameters, all collected ticks were classified as I. ricinus. Identification of the developmental stage resulted in 573 adults (288 females and 285 males) and 1527 nymphs.

\section{Borrelia infections in ticks in 2015}

In 2015 , a total of $24.1 \%(505 / 2100)$ of ticks were infected with Borrelia spp., subdivided into $35.4 \%$ adults (203/573), thereof $38.5 \%$ females (111/288) and 32.3\% males $(92 / 285)$, as well as $19.8 \%$ nymphs $(302 / 1527)$. Infection rates of different stages resulted in significant differences between nymphs $v s$ females as well as males $\left(\chi^{2}=21.34-47.48, d f=1, P<0.0001\right)$.

Concerning seasonal variations, no significant differences were detected between individual sampling months. Conversely, tick infection rates varied significantly between different sampling sites. The highest infection rate of 31.4\% (66/ 210) was determined in the inner urban park "Georgengarten" which differed significantly from sampling sites "Annateiche" $\left(14.8 \%, 31 / 210 ; X^{2}=15.50, d f=1 P<0.0001\right)$ and "Ricklinger Teiche" $\left(15.2 \%, 32 / 210 ; \chi^{2}=14.49, d f=1\right.$ $P=0.0001)$, which showed the lowest determined tick infection rates. Detailed information about seasonal distribution of Borrelia infected tick stages is provided in Table 1, information about local distribution is provided in Table 2.

\section{Tick infections with Borrelia in 2015 vs 2010 and 2005}

Over the entire monitoring period of ten years, total tick infection rates as well as infection rates in different stages remained consistent (Fig. 1), which was also observed for different sampling sites when comparing 2010 [9] to 2015. Concerning local infection rates, a significant decrease was determined at "Ricklinger Teiche" between 2005 and 2015 (31.7 vs 15.2\%; $\chi^{2}=17.30, d f=1$, $P<0.0001)$. Contrary, infection rates significantly increased between 2005 and 2015 at "Georgengarten" (19.3 vs $\left.31.4 \% ; \chi^{2}=10.06, d f=1, P=0.0015\right)$ as well as "Maschpark" (14.3 vs 27.7\%; $\chi^{2}=7.565, d f=1 P=0$. 0059). Furthermore, significantly higher Borrelia infection rates $\left(\chi^{2}=11.09, d f=1, P=0.0009\right)$ were 
Table 1 Seasonal distribution of Borrelia spp. infected Hanoverian ticks (positives/total ticks) in 2015

\begin{tabular}{lllllllll}
\hline Seasonal distribution & April & May & June & July & August & September & October & Total \\
\hline Adults & $27 / 95$ & $33 / 97$ & $42 / 95$ & $25 / 80$ & $29 / 75$ & $28 / 72$ & $19 / 59$ & $203 / 573$ \\
$(\%)$ & $(28.4)$ & $(34.0)$ & $(44.2)$ & $(31.3)$ & $(38.7)$ & $(38.9)$ & $(32.2)$ & $(35.4)^{\mathrm{a}}$ \\
Adult males & $13 / 53$ & $12 / 48$ & $21 / 51$ & $10 / 42$ & $18 / 37$ & $11 / 28$ & $7 / 27$ & $92 / 285$ \\
$(\%)$ & $(24.5)$ & $(25.0)$ & $(41.2)$ & $(23.8)$ & $(48.6)$ & $(39.3)$ & $(25.9)$ & $(32.3)^{\mathrm{a}}$ \\
Adult females & $14 / 43$ & $21 / 49$ & $21 / 44$ & $15 / 38$ & $11 / 38$ & $17 / 44$ & $12 / 32$ & $111 / 288$ \\
$(\%)$ & $(32.6)$ & $(42.9)$ & $(47.7)$ & $(39.5)$ & $(28.9)$ & $(38.6)$ & $(37.5)$ & $(38.5)^{\mathrm{a}}$ \\
Nymphs & $49 / 205$ & $39 / 203$ & $52 / 205$ & $40 / 220$ & $32 / 225$ & $48 / 228$ & $41 / 241$ & $302 / 1527$ \\
$(\%)$ & $(23.9)$ & $(19.2)$ & $(25.4)$ & $(18.2)$ & $(14.2)$ & $(21.1)$ & $(17.0)$ & $(19.7)^{\mathrm{a}}$ \\
Total & $76 / 300$ & $72 / 300$ & $94 / 300$ & $65 / 300$ & $61 / 300$ & $76 / 300$ & $61 / 300$ & $505 / 2100$ \\
$(\%)$ & $(25.3)$ & $(24.0)$ & $(31.3)$ & $(21.7)$ & $(20.3)$ & $(25.3)$ & $(20.3)$ & $(24.1)$ \\
\hline
\end{tabular}

${ }^{\mathrm{a}}$ Significantly higher infection rates in adults (females and males) vs nymphs $(P<0.05)$

determined in October 2010 (32.7\%) [9] than in October 2015 (20.3\%). Comparison of seasonal data over the tenyear period revealed significantly higher infection rates in September/October 2010 [9] than in September/October 2005 [31] (27.5 vs 16.3\%; $\chi^{2}=9.244, d f=1, P=0$. 0024). Detailed comparison of the seasonal distribution in 2005, 2010 and 2015 is depicted in Fig. 2.

\section{Design of the Borrelia miyamotoi probe MIYA}

The obtained sequence of B. miyamotoi strain HT 31 showed 99\% identity with B. miyamotoi from Japan and $95 \%$ identity with sequences from the USA. The newly designed probe MIYA targeting the hydrolase-23S rRNA IGS showed $100 \%$ sequence identity with all available sequences contrary to the previously used RFLNE probe $[9,10,12]$ designed by Gern et al. [29] (Fig. 3). Comparison of the 25 bp probe MIYA with published sequences of the hydrolase-23S IGS region of other relapsing fever Borrelia and the 5S-23S IGS region of B. burgdorferi (s. $l$.) as well as related species showed no similarities to respective loci (data not shown). Additionally, no crossreactions with DNA from different geno-species of the B. burgdorferi (s.l.) complex, the relapsing fever borreliae B. duttonii, B. hermsii and B. recurrentis (Fig. 4) as well as Treponema phagedenis and Leptospira spp. (data not shown) were observed.

\section{Borrelia geno-species identification in ticks}

Borrelia geno-species differentiation by RLB was successful in $52.7 \%(266 / 505)$ of qPCR Borrelia-positive ticks. Sensitivity of conducted RLB was dependent on the number of 5S-23S IGS copies, resulting in 100\% (15/ 15) differentiation of samples containing $\geq 10^{4}$ copies, 91.7\% (55/60) of samples containing $\geq 10^{3}$ copies, $84.6 \%$ $(121 / 143)$ of samples containing $\geq 10^{2}$ copies and $50.4 \%$ (57/113) of samples containing $\geq 10^{1}$ copies. Of samples containing $\leq 10$ copies, only $10.3 \%(18 / 174)$ were differentiated. Borrelia afzelii was the most frequently occurring geno-species $(16.8 \%$; $85 / 505)$ followed by B. garinii/B. bavariensis as well as B. valaisiana (each $10.7 \%$;

Table 2 Local distribution of Borrelia spp. infected Hanoverian ticks (positives/total ticks) in 2015

\begin{tabular}{|c|c|c|c|c|c|c|c|c|c|c|}
\hline $\begin{array}{l}\text { Local } \\
\text { distribution }\end{array}$ & Mecklenheide & $\begin{array}{l}\text { Große } \\
\text { Heide }\end{array}$ & $\begin{array}{l}\text { Misburger } \\
\text { Wald }\end{array}$ & Annateiche & $\begin{array}{l}\text { Seelhorster } \\
\text { Wald }\end{array}$ & $\begin{array}{l}\text { Ricklinger } \\
\text { Teiche }\end{array}$ & $\begin{array}{l}\text { Bornumer } \\
\text { Holz }\end{array}$ & Georgengarten & Eilenriede & Maschpark \\
\hline Adults & $27 / 72$ & $39 / 84$ & $6 / 20$ & $7 / 40$ & $36 / 93$ & $13 / 40$ & $27 / 74$ & $14 / 36$ & $17 / 91$ & $7 / 23$ \\
\hline (\%) & (37.5) & $(46.2)$ & $(30.0)$ & $(17.5)$ & $(38.7)$ & $(32.5)$ & $(36.5)$ & $(38.9)$ & $(18.7)$ & $(30.4)$ \\
\hline Adult males & $13 / 35$ & $14 / 37$ & $1 / 11$ & $2 / 21$ & $24 / 45$ & $5 / 23$ & $14 / 39$ & $6 / 19$ & $11 / 46$ & $2 / 9$ \\
\hline (\%) & $(37.1)$ & $(37.8)$ & $(9.1)$ & $(9.5)$ & (53.3) & $(21.7)$ & $(35.9)$ & (31.6) & $(23.9)$ & $(22.2)$ \\
\hline $\begin{array}{l}\text { Adult } \\
\text { females }\end{array}$ & $14 / 37$ & $25 / 47$ & $5 / 9$ & $5 / 19$ & $12 / 48$ & $8 / 17$ & $13 / 35$ & $8 / 17$ & $16 / 45$ & $5 / 14$ \\
\hline (\%) & (37.8) & $(53.2)$ & $(55.6)$ & $(26.3)$ & $(25.0)$ & $(47.1)$ & $(37.1)$ & $(47.1)$ & (35.6) & $(35.7)$ \\
\hline Nymphs & $34 / 138$ & $24 / 126$ & 43/190 & $24 / 170$ & $20 / 117$ & 19/170 & $25 / 136$ & $52 / 174$ & $14 / 119$ & $47 / 187$ \\
\hline (\%) & $(24.6)$ & (19.0) & $(22.6)$ & (14.1) & $(17.1)$ & $(11.2)$ & $(18.4)$ & $(29.9)$ & (11.8) & $(25.1)$ \\
\hline Total & $61 / 210$ & $63 / 210$ & $49 / 210$ & $31 / 210$ & $56 / 210$ & $32 / 210$ & $52 / 210$ & $66 / 210$ & $41 / 210$ & $54 / 210$ \\
\hline (\%) & $(29.0) b$ & $(30.0) \mathrm{b}$ & $(23.3)$ & (14.8)b & $(26.7)$ & $(15.2) \mathrm{b}$ & $(24.8)$ & $(31.4) b$ & (19.5) & $(25.7)$ \\
\hline
\end{tabular}

"Significantly higher infection rates in "Mecklenheide", "Große Heide" and "Georgengarten" vs "Annateiche" and "Ricklinger Teiche" $(P<0.05)$ 


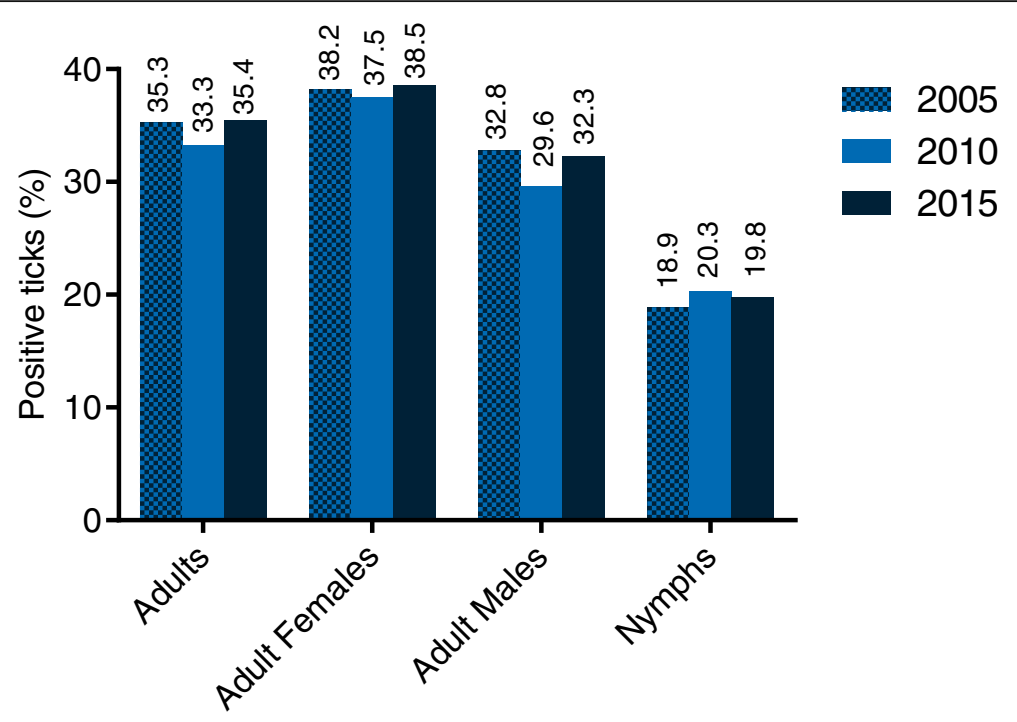

Fig. 1 tick infection rates with Borrelia spp. over the ten-year monitoring period (2005 [11] vs 2010 [9] vs 2015)

54/505) B. burgdorferi (s.s.) (6.5\%; 33/505), B. spielmanii (3.0\%; 15/505), B. bissettiae (1.6\%; 8/505) and B. lusitaniae $(0.4 \% ; 2 / 505)$. The relapsing fever species $B$. miyamotoi was detected in $8.9 \%(45 / 505)$ of Borrelia-positive ticks. Regarding multiple-infections, the majority of ticks (46. $5 \%$; 235/505) were mono-infected, 5.5\% (28/505) were double-infected and $0.6 \%$ (3/505) were triple-infected.

Out of 54 B. garinii/B. bavariensis RLB positive tick samples, 43 were successfully identified by Sanger sequencing. Of these, $97.7 \%(42 / 43)$ were identified as $B$. garinii and $2.3 \%(1 / 42)$ as B. bavariensis. Out of the 33 B. burgdorferi (s.s.)/B. carolinensis RLB-positive tick samples, 26 (78.8\%) were identified by Sanger sequencing. Of these all $(26 / 26)$ were identified as $B$. burgdorferi (s.s.) and none $(0 / 26)$ as $B$. carolinensis. Out of the 8 B. bissettiae/B. kurtenbachii RLB-positive tick samples, none $(0 / 8)$ were successfully identified by Sanger sequencing. Detailed information on geno-species distribution in Borrelia-positive ticks is given in Table 3.

\section{Borrelia geno-species in 2015 vs 2010 and 2005}

The number of tick mono-infections with $B$. afzelii, and $B$. spielmanii decreased significantly from $2010[9,10]$ to 2015 (B. afzelii: 30.9 vs 16.8\%, $\chi^{2}=26.02, d f=1, P<0.0001 ; B$. spielmanii: 10.9 vs $\left.3.0 \%, \chi^{2}=23.13, d f=1, P<0.0001\right)$. In the study year $2015, B$. afzelii was the predominant genospecies, followed by B. garinii/B. bavariensis and B. valaisiana (each 10.7\%, see Table 3). In 2010, B. afzelii as the
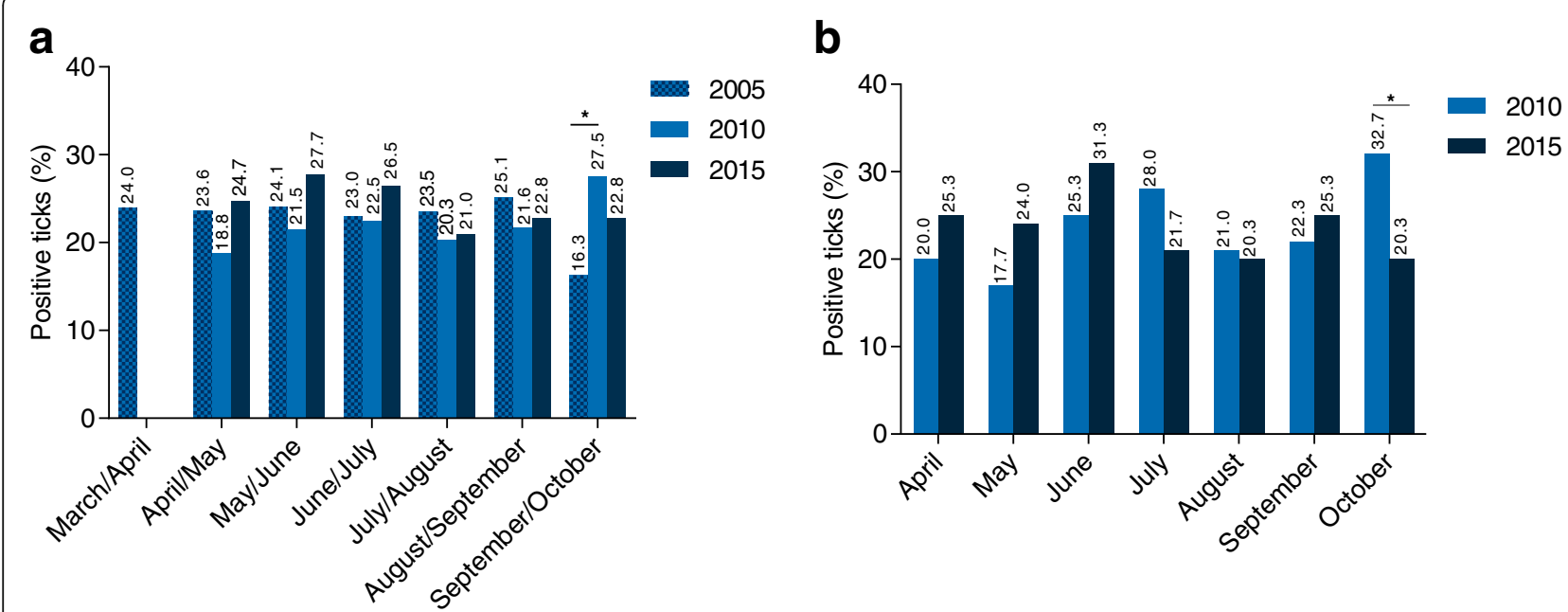

Fig. 2 seasonal distribution of Borrelia-positive ticks in a 2005-2015 (seasonal data of individual sampling months was not obtained in 2005, wherefore data obtained in 2010 and 2015 was modified accordingly) $[9,31]$ and b 2010 [9] vs 2015. ${ }^{*} P \leq 0.05$ 


\begin{tabular}{|c|c|c|c|}
\hline \multicolumn{2}{|c|}{ Species/accession no. } & \multicolumn{2}{|c|}{ Sequence } \\
\hline & & & $\begin{array}{ll}\text { B5S-Bor } & \text { BMIYA-For }\end{array}$ \\
\hline Bmiya* & HT 31 & 1 & ---------tgaaattattgagggtaagtgaacaattttaggattaatgatgtttttac \\
\hline Bmiya & GQ387038 & 1 & --------tgcaattattgagggcaaatgaacgattttaggattaatgatgtttttac \\
\hline Bmiya & $\mathrm{CP} 004217$ & 1 & gtaggttatttgaaattattgagggtaagtgaacaattttaggattaatgatgtttttac \\
\hline Bmiya & CP017126 & 1 & gtaggttatttgaagttagtgagggcaaatggacgattttaggattaatgatattgttac \\
\hline Bmiya & AY531879 & 1 & gtaggttatttgaagttagtgagggcaaatggacgattttaggattaatgatattgttac \\
\hline Bmiya & CP010308 & 1 & gtaggttatttgaagttagtgagggcaaatggacgattttaggattaatgatattgttac \\
\hline Bmiya & $\mathrm{CP} 006647$ & 1 & $\begin{array}{l}\text { gtaggttatttgaagttagtgagggcaaatggacgattttaggattaatgatattgttac } \\
\text { RFLNE probe }\end{array}$ \\
\hline Bmiya* & HT 31 & 51 & ctgtaagatttata-cctggcattgctcaatatggttagtgtgatttaatttttgaaaa \\
\hline Bmiya & GQ387038 & 51 & ctgtaagatttatatcctagcattgatcaa--tggatagtgtgatttaatttttgaaaaa \\
\hline Bmiya & $\mathrm{CP} 004217$ & 61 & ctgtaagatttata-cctggcattgctcaatatggatagtgtgatttaatttttgaaaa \\
\hline Bmiya & CP017126 & 61 & ctataagatttata-tctagcattgagcaa--tggatagtgtgatttaagttttgaaaa \\
\hline Bmiya & AY531879 & 61 & ctataagatttata-tctagcattgagcaa--tggatagtgtgatttaagttttgaaaaa \\
\hline Bmiya & CP010308 & 61 & ctataagatttata-tctagcattgagcaa--tggatagtgtgatttaagttttgaaaa \\
\hline Bmiya & CP006647 & 61 & $\begin{array}{l}\text { ctataagatttata-tctagcattgagcaa--tggatagtgtgatttaagttttgaaaa } \\
\text { MIYA probe }\end{array}$ \\
\hline Bmiya* & HT 31 & 110 & ttatttagtgaaagttcgataatagtattgacatagtaaagttaaaatatttattagt \\
\hline Bmiya & GQ387038 & 109 & ttatttagtgaaagttcaataatagtattgacatagtaaagttaaaatatatattaat \\
\hline Bmiya & CP004217 & 120 & ttatttagtgaaaagttcaataatagtattgacatagtaaagttaaaatatatattagt \\
\hline Bmiya & $\mathrm{CP} 017126$ & 118 & ttatttagtgaaagttcaataatagtattgacatagtaaagttaaaatatatattagt \\
\hline Bmiya & AY531879 & 118 & ttatttagtgaaagttcaataatagtattgacatagtaaagttaaaatatatattagt \\
\hline Bmiya & CP010308 & 118 & ttatttagtgaaagttcaataatagtattgacatagtaaagttaaaatatatattagt \\
\hline Bmiya & CP006647 & 118 & ttatttagtgaaaagttcaataatagtattgacatagtaaagttaaaatatatattagt \\
\hline Bmiya* & HT 31 & 170 & ttatgttgtataaacgattggcaaattagagacggaagataaaatatggtcaaagtaat \\
\hline Bmiya & $\mathrm{GQ387038}$ & 169 & ttatgttgtataaacgattggcaaattagagacggaagataaaatatggtcaaagtaat \\
\hline Bmiya & $\mathrm{CP} 004217$ & 180 & ttatgttgtataaacgattggcaaattagagacggaagataaaatatggtcaaagtaat \\
\hline Bmiya & CP017126 & 178 & ttatgttgtataaacgattggcaaattagagacggaagataaaatatggtcaaagtaat \\
\hline Bmiya & AY531879 & 178 & ttatgttgtataaacgattggcaaattagagacggaagataaaatatggtcaaagtaat \\
\hline Bmiya & $\mathrm{CP} 010308$ & 178 & ttatgttgtataaacgattggcaaattagagacggaagataaaatatggtcaaagtaat \\
\hline Bmiya & CP006647 & 178 & ttatgttgtataaacgattggcaaattagagacggaagataaaatatggtcaaagtaat \\
\hline Bmiya* & HT 31 & 230 & aaagagtctatggtggatgcctaggagctttaaggcgaagaaggtcgtggtaagctgcga \\
\hline Bmiya & GQ387038 & 229 & aaagagtctatggtggatgcctaggagctttaaggcgaagaaggtcgtggtaagctgcga \\
\hline Bmiya & $\mathrm{CP} 004217$ & 240 & aaagagtctatggtggatgcctaggagctttaaggcgaagaaggtcgtggtaagctgcga \\
\hline Bmiya & $\mathrm{CP} 017126$ & 238 & aaagagtctatggtggatgcctaggagctttaaggcgaagaaggtcgtggtaagctgcga \\
\hline Bmiya & AY531879 & 238 & aaagagtctatggtggatgcctaggagctttaaggcgaagaaggtcgtggtaagctgcga \\
\hline Bmiya & CP010308 & 238 & aaagagtctatggtggatgcctaggagctttaaggcgaagaaggtcgtggtaagctgcga \\
\hline Bmiya & CP006647 & 238 & aaagagtctatggtggatgcctaggagctttaaggcgaagaaggtcgtggtaagctgcga \\
\hline Bmiya* & HT 31 & 290 & aaagcttgggggagaagcaaacattttacgatcccgagatcaccgaatggagtaatccaa \\
\hline Bmiya & GQ387038 & 289 & aaagcttgggggagaagcaaacattttatgatcccgaggtcaccgaatggagtaatccaa \\
\hline Bmiya & $\mathrm{CP} 004217$ & 300 & aaagcttgggggagaagcaaacattttacgatcccgagatcaccgaatggagtaatccaa \\
\hline Bmiya & CP017126 & 298 & aaagcttgggggagaagcaaacattttatgatcccgagatcaccgaatggagtaatccaa \\
\hline Bmiya & AY531879 & 298 & aaagcttgggggagaagcaaacattttatgatcccgagatcaccgaatggagtaatccaa \\
\hline Bmiya & $\mathrm{CP} 010308$ & 298 & aaagcttgggggagaagcaaacattttatgatcccgagatcaccgaatggagtaatccaa \\
\hline Bmiya & CP006647 & 298 & aaagcttgggggagaagcaaacattttatgatcccgagatcaccgaatggagtaatccaa \\
\hline Bmiya* & HT 31 & 350 & ttagctagatgctaattaattactacgtaagtagtaaaggctataccagggg------ \\
\hline Bmiya & GQ387038 & 349 & ttagttagatgctaattatttactacgtaagtagtaaaagcta-accagggg-- \\
\hline Bmiya & $\mathrm{CP} 004217$ & 360 & ttagctagatgctaattatttactacgtaagtagtaaaggctataccaggggaagtgaaa \\
\hline Bmiya & CP017126 & 358 & ttagctagatgctaattatttactacgtaagtagtaaaggctataccaggggaagtgaaa \\
\hline Bmiya & AY531879 & 358 & ttagctagatgctaattatttactacgtaagtagtaaaggctataccaggggaagtgaaa \\
\hline Bmiya & CP010308 & 358 & ttagctagatgctaattatttactacgtaagtagtaaaggctataccaggggaagtgaaa \\
\hline Bmiya & $\mathrm{CP} 006647$ & 358 & ttagctagatgctaattatttactacgtaagtagtaaaggctataccaggggaagtgaaa \\
\hline & & & 3S-Bor \\
\hline Bmiya* & HT 31 & & \\
\hline Bmiya & GQ387038 & & \\
\hline Bmiya & $\mathrm{CP} 004217$ & 420 & catctaagtaccctga \\
\hline Bmiya & CP017126 & 418 & catctaagtaccctga \\
\hline Bmiya & AY531879 & 418 & catctaagtaccctga \\
\hline Bmiya & CP010308 & 418 & catctaagtaccctga \\
\hline Bmiya & CP006647 & 418 & catctaagtaccetga \\
\hline
\end{tabular}

Fig. 3 Alignment of the hydrolase-23S rRNA IGS sequence of Borrelia miyamotoi from different origins. Substitutions and insertions/deletions are located to the loci of the RFLNE probe (blue) [28], whereas probe MIYA (red) displays 100\% identity with aligned sequences

predominant geno-species was followed by B. garinii/B. bavariensis and B. spielmanii $[9,10]$, whereas in $2005, B$. garinii/B. bavariensis was the predominant geno-species, followed by B. afzelii and B. spielmanii [11]. A significant decrease in double-infections between 2015 vs 2010 (5.5\% vs $\left.13.4 \%, \chi^{2}=17.08, d f=1, P<0.0001\right)[9,10]$ and 2005 (24.3\%, $\chi^{2}=55.01, d f=1, P<0.0001$ ) [11] was determined, whereas numbers of triple-infections remained consistent between years. Furthermore, no quadruple-infection was determined in 2015 contrary to $2010[9,10]$. 


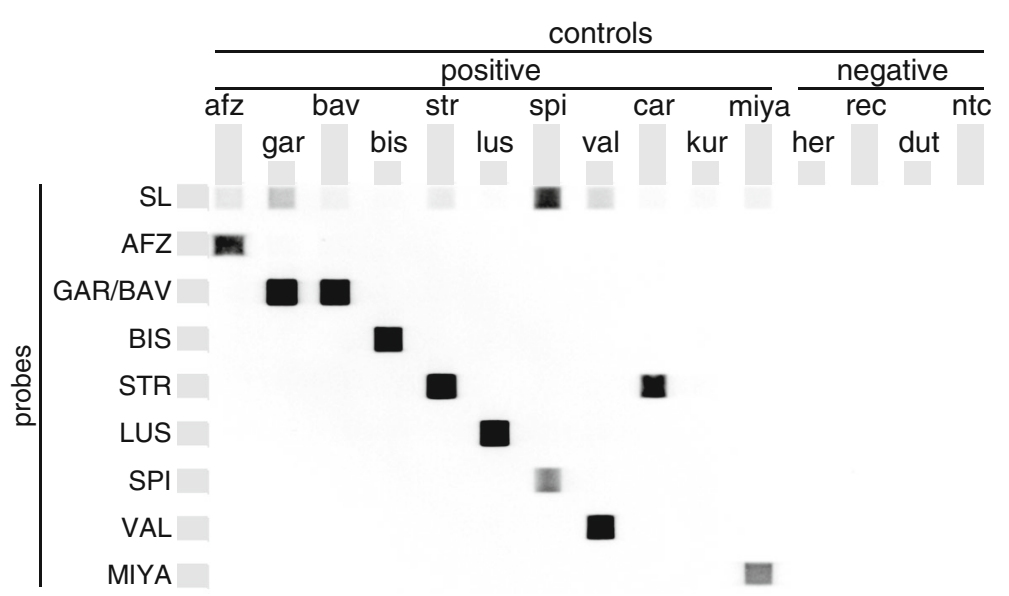

Fig. 4 Exemplary Reverse Line Blot (RLB) image, showing the specific detection of amplified B. miyamotoi (miya) DNA by the newly designed probe MIYA. Additionally, detection of B. burgdorferi (s.l.) geno-species by different probes (SL, B. burgdorferi (s.l.); AFZ, B. afzelii; GAR/BAV, B. garinii/B. bavariensis; BIS, B. bissettiae; STR, B. burgdorferi (s.s.); LUS, B. lusitaniae; SPI, B. spielmanii; VAL, B. valaisiana) is shown. Controls were DNA amplification products of $B$. afzelii (afz), B. garinii (gar), B. bavariensis (bav), B. bissettiae (bis), B. burgdorferi (s.s.) (str), B. lusitaniae (lus), B. spielmanii (spi), B. valaisiana (val), B. carolinensis (car), B. kurtenbachii (kur), B. hermsii (her), B. recurrentis (rec) and B. duttonii (dut). Abbreviation: ntc, no-template control

Tick co-infections with Borrelia and Rickettsiales in 2015 vs 2010 and 2005

In $2015,10.9 \%(229 / 2100)$ of ticks were co-infected with Rickettsia species, whereas co-infections with $A$. phagocytophilum were detected in 1.1\% (23/2100) of ticks. Furthermore, $0.6 \%(12 / 2100)$ of ticks were infected with all three pathogens (Table 4). Significantly increased numbers of Borrelia/Rickettsia spp. (10.9 vs $\left.7.3 \% ; X^{2}=16.20, d f=1, P<0.0001\right)$ as well as Borrelia/A. phagocytophilum (1.1 vs $0.3 \% ; \chi^{2}=7.554$, $d f=1, P=0.006)$ co-infected ticks were detected in 2015 vs 2010 [9, 10], whereas consistent co-infection rates were found between 2015 vs 2005. Concerning triple-infections, no significant differences were found between study years.

\section{Discussion}

Besides Lyme borreliae of the B. burgdorferi (s.l.) complex, the relapsing fever agent $B$. miyamotoi has frequently been detected in I. ricinus in Europe during the last decade and human pathogenicity has been observed $[6,32]$. As both pathogens utilize I. ricinus as vector, monitoring of tick infection rates is an indispensable part of a public health risk assessment. To date, no data about $B$. miyamotoi tick infection rates in northern Germany are available wherefore an RLB-protocol was established, enabling specific detection of B. miyamotoi in addition to B. burgdorferi (s.l.) geno-species differentiation. By comparing current data with those obtained in 2010 and 2005, the first 10-year follow-up monitoring of Borrelia tick infections in Germany was performed. Over

Table 3 Reverse Line Blot results on B. burgdorferi (s.l.) geno-species and B. miyamotoi distribution in infected ( $n=505)$ ticks in 2015

\begin{tabular}{|c|c|c|c|c|c|c|c|}
\hline $\begin{array}{l}\text { Total no. } \\
\text { of infections }\end{array}$ & $\begin{array}{l}\text { No. of } \\
\text { infected } \\
\text { ticks (\%) }\end{array}$ & $\begin{array}{l}\text { Mono- } \\
\text { infection }\end{array}$ & $\begin{array}{l}\text { No. of } \\
\text { infected } \\
\text { ticks (\%) }\end{array}$ & $\begin{array}{l}\text { Double- } \\
\text { infection }\end{array}$ & $\begin{array}{l}\text { No. of } \\
\text { infected } \\
\text { ticks (\%) }\end{array}$ & Triple-infection & $\begin{array}{l}\text { No. of } \\
\text { infected } \\
\text { ticks (\%) }\end{array}$ \\
\hline Baf & $85(16.8)$ & Baf & $70(13.9)$ & $B a f+B s s / B c a r$ & $7(1.4)$ & $B a f+B b i / B k u+B s s / B c a$ & $3(0.6)$ \\
\hline $\mathrm{Bga} / \mathrm{Bba}$ & $54(10.7)$ & Bva & $47(9.3)$ & $B g a / B b a+B v a$ & $6(1.2)$ & & \\
\hline Bva & $54(10.7)$ & $B g a / B b a$ & $46(9.1)$ & $B a f+B g a / B b a$ & $1(0.2)$ & & \\
\hline$B s s / B c a$ & $33(6.5)$ & $B s s / B c a$ & $19(3.8)$ & $B g a / B b a+B l u$ & $1(0.2)$ & & \\
\hline Bsp & $15(3.0)$ & Bsp & $13(2.6)$ & $B a f+B s p$ & $1(0.2)$ & & \\
\hline Bbi/Bku & $8(1.6)$ & Blu & $1(0.2)$ & $B a f+B m i$ & $3(0.6)$ & & \\
\hline Blu & $2(0.4)$ & Bbi/Bku & $0(0.0)$ & $B s p+B m i$ & $1(0.2)$ & & \\
\hline Bmi & $45(8.9)$ & Bmi & $39(7.7)$ & $B v a+B m i$ & $1(0.2)$ & & \\
\hline No geno-species determined & $239(47.3)$ & & & $B s s / B c a+B m i$ & $1(0.2)$ & & \\
\hline
\end{tabular}

Abbreviations: Baf, B. afzelii; Bva, B. valaisiana; Bss, B. burgdorferi (s.s.); Bca, B. carolinensis; Bga, B. garinii; Bba, B. bavariensis; Bsp, B. spielmanii; Blu, B. lusitaniae; Bbi, B. bissettiae; Bku, B. kurtenbachii; Bmi, B. miyamotoi 
Table 4 Coinfections with B. burgdorferi (s.l.) and Rickettsiales in Hanoverian I. ricinus in 2015

\begin{tabular}{lllllll}
\hline & $\begin{array}{l}\text { No. of } \\
\text { collected ticks }\end{array}$ & $\begin{array}{l}\text { No. of B. burgdorferi } \\
(\text { s.l.) positive ticks }\end{array}$ & $\begin{array}{l}\text { Total } \\
\text { coinfections }\end{array}$ & $\begin{array}{l}\text { Rickettsia spp. } \\
\text { coinfections }\end{array}$ & $\begin{array}{l}\text { A. phagocytophilum } \\
\text { coinfections }\end{array}$ & $\begin{array}{l}\text { Coinfections with } \\
\text { Rickettsia spp. and } \\
\text { A. phagocytophilum }\end{array}$ \\
\hline Adult & 573 & No. (\%) & No. (\%) & No. (\%) & No. (\%) & No. (\%) \\
Males & 285 & $203(35.4)$ & $113(20.0)$ & $92(16.1)$ & $14(2.4)$ & $7(1.22)$ \\
Females & 288 & $92(32.3)$ & $49(17.2)$ & $40(14.0)$ & $5(1.8)$ & $4(1.4)$ \\
Nymphs & 1527 & $111(38.5)$ & $64(22.2)$ & $52(18.1)$ & $9(3.1)$ & $3(1.0)$ \\
All stages & 2100 & $302(19.8)$ & $151(9.8)$ & $137(9.0)$ & $9(0.6)$ & $5(0.33)$ \\
\hline
\end{tabular}

this period, infection rates were consistent in all investigated tick stages, but varied at three sampling sites. Furthermore, geno-species distribution showed significant differences. As certain geno-species are reliant on specific reservoir hosts (e.g. rodents or birds), observed variations may reflect alterations in the availability of reservoir host species between study years.

In 2015, approximately one quarter of examined ticks was infected with Borrelia spp., with adult ticks being significantly more often infected than nymphs. This was also observed in the studies in 2005 and 2010 and is explainable by efficient transstadial transmission [33] as well as an increased probability to acquire spirochetes by an extra blood meal taken during the nymphal stage [9]. Local tick infection also differed significantly within the city of Hanover in 2015, ranging from a minimum of $14.8 \%$ at "Annateiche", an extensive forest area, to a maximum of $31.4 \%$ at "Georgengarten", an inner urban public park. As high Borrelia prevalences were also found at other sampling sites characterized by extensive forest areas, respective infection rates are most likely explainable by local abundance of infected reservoir hosts. Local abundance of reservoir hosts could possibly be influenced by landscape features and high frequency of visitors, as the increase in infection rates between 2005 and 2015 was only observed for urban parks. Presumably, intense usage and cultivation of landscape areas may lead to an increased density of e.g. bank voles or other small rodents, serving as suitable reservoir hosts. In contrast, wildlife fauna in forest areas may be more diverse and include species such as roe deer or wild boar which are unsuitable Borrelia hosts, possibly leading to a dilution effect [34]. Varying tick infection rates at individual sampling sites over the ten-year monitoring period again indicate an altered distribution of Borrelia spp. infections in reservoir hosts [33]. Differences in (micro)climatic conditions as well as the local vegetation between study years probably contribute to the altered annual Borrelia spp. infection rates by affecting both, reservoir host and questing tick abundance.

Contrary to local differences in 2015, Borrelia spp. infection rates showed no seasonal variations. When comparing seasonal data of 2015 vs 2010, significantly decreased infection rates were found for October 2015. Climatic differences, like a possibly more moderate and humid autumn in 2010, may account for this difference between the years, since they may have an impact on reservoir host availability besides tick density and activity [1].

Borrelia geno-species differentiation by RLB was successful in $52.7 \%$ of infected ticks. Compared to the study in $2010[9,10]$, the RLB procedure in 2015 was modified to enable successful $B$. miyamotoi amplification. RLB hybridisation (Fig. 4) showed that available probes for differentiation of B. burgdorferi (s.l.) geno-species generally recognise all geno-species occurring in Europe (however, subsequent sequencing is needed to differentiate some geno-species due to cross-reactions) with the limitation that detection of $B$. finlandensis was not tested as no positive control strain could be included. Even though no specific RLB-probe for B. finlandensis is available, it seems unlikely that undetermined tick samples were infected with that particular geno-species as it was found in only one I. ricinus in Europe previously [35]. Undetermined samples rather result from the lower RLB sensitivity compared to qPCR, which detects one target copy in the reaction set-up $[9,11]$, whereas the RLB identified the infecting geno-species in only $10.3 \%$ of samples containing $\leq 105 S-23 S$ rRNA IGS copies in the recent study. Despite consistent total Borrelia infection rates, RLB revealed variations in distribution of different $B$. burgdorferi (s.l.) geno-species between 2015 and 2010. Interestingly, a significant decrease in the rodent associated geno-species B. afzelii and B. spielmanii $[36,37]$ was determined in 2015 vs 2010. The decrease may be based on a correlation between available vertebrate reservoir hosts and distribution of Borrelia species in ticks $[37,38]$. Such correlations may also explain higher $B$. garinii infection rates in 2005 and 2015 vs 2010 [9-11]. Generally, it should be noted that geno-species differentiation in 2005 was achieved by conventional PCR instead of RLB as performed in 2010 [9] and in the current study. Regarding multiple-infections, doubleinfections significantly decreased in 2015 compared to 2010 [9]. In 2010, double-infections were mainly caused by $B$. afzelii and B. spielmanii [9], and decreased 
occurrence of named geno-species in 2015 may have consequently resulted in decreased numbers of total double-infections. B. afzelii was found in all determined triple-infections in the present study and was frequently found in triple-infections in 2010 [9]. However, tripleinfections appear to be a consistently rare event as ticks are required to feed from multiple hosts, multipleinfected hosts or acquire an infection via co-feeding with (multiple-) infected ticks [5, 39]. The absence of quadruple-infections in 2015 was also not unexpected, as detection was only described once in 2010 [9] but not in 2005 [11].

A total of $8.9 \%(45 / 505)$ Borrelia-positive ticks were infected with $B$. miyamotoi, representing the first available prevalence data for northern Germany. The developed B. miyamotoi specific RLB-probe presumably enables detection of worldwide occurring $B$. miyamotoi strains (Fig. 3) and is therefore highly valuable. As $B$. miyamotoi may cause mild as well as severe disease in humans $[6,21,22,40]$, the occurrence in ticks in inner urban recreation areas should be considered as a risk to public health and monitored accordingly, in particular as $8.9 \%$ of ticks identified for their Borrelia genospecies were infected with this pathogen. Even though the presence of $B$. miyamotoi was not investigated in previous studies conducted in Hannover, the detection of Borrelia-positive I. ricinus larvae in 2010 [9] indicate its occurrence due to highly efficient transovarial transmission in ticks contrary to inefficient transovarial transmission of B. burgdorferi (s.l.) [9, 41]. Furthermore, successful transmission of $B$. miyamotoi from infected $I$. ricinus larvae to vertebrates has been shown in in vivo experiments, indicating an infection risk from larval tick bites [42]. Therefore, further examination of larval I. ricinus populations would aid in assessing the risk of transmission to humans. In this context, it is essential to sensitise clinicians to consider B. miyamotoi as a causative agent when treating patients with unspecific feverish infections as $B$. miyamotoi induces rather unspecific symptoms or may mimic other tick-borne diseases like human granulocytic anaplasmosis (HGA) [43]. Mimicking HGA may occur frequently as about $14.6 \%$ of patients with suspected but serologically unconfirmed HGA were determined anti-B. miyamotoi antibody positive in the Netherlands [44].

Concerning co-infections with Rickettsiales, increased co-infection rates with Rickettsia spp. in 2015 may above all be based on generally increased Rickettsia spp. infection rates in 2015 vs 2010 (50.8 vs 26.2\%) [23, 25, 26]. Co-infections of Borrelia spp. with A. phagocytophilum also increased from 2010 to 2015, but not from 2005 to 2015 , although tick infection rates of both pathogens remained consistent $[17,23,25,26]$. Further monitoring regarding co-infection rates are desired as investigations of experimentally A. phagocytophilum/B. burgdorferi (s. s.) co-infected mice showed increased bacterial load and pathogen transmission to the tick vector as well as an increase in severity of Lyme disease compared to monoinfections $[45,46]$. Therefore, even though numbers of Borrelia/A. phagocytophilum co-infected ticks are generally low, potentially enhanced severity in Lyme disease manifestation should increase awareness of pathogen transmission from co-infected ticks.

\section{Conclusions}

The RLB protocol established in the present study enables specific detection of $B$. miyamotoi besides differentiation of common European B. burgdorferi (s.l.) geno-species, resulting in the first detection of B. miyamotoi in I. ricinus in northern Germany. Although the overall determined Borrelia tick infection rate remained consistent over the 10-year monitoring period, shifts in occurrence of human pathogenic geno-species underline the importance of detailed analyses for diagnostic and research purposes as well as for a public health risk assessment.

\section{Abbreviations}

RLB: Reverse line blot

\section{Availability of data and materials}

All data generated or analyzed during this study are included in this published article.

\section{Authors' contributions}

KB collected the ticks. KB, MKR and DJ carried out the experimental work. VF provided Borrelia control DNA. KB drafted the manuscript. CS designed and coordinated the study. All authors participated in data analysis and interpretation, and read and approved the final manuscript.

Ethics approval and consent to participate Not applicable.

Competing interests

The authors declare that they have no competing interests.

\section{Publisher's Note}

Springer Nature remains neutral with regard to jurisdictional claims in published maps and institutional affiliations.

\section{Author details}

${ }^{1}$ Institute for Parasitology, Centre for Infection Medicine, University of Veterinary Medicine Hannover, Buenteweg 17, 30559 Hanover, Germany. ${ }^{2}$ Immunology Unit and Research Center for Emerging Infections and Zoonoses, University of Veterinary Medicine Hannover, Buenteweg 17, 30559 Hanover, Germany. ${ }^{3}$ German National Reference Centre for Borrelia, Oberschleißheim, Germany.

Received: 5 February 2018 Accepted: 30 April 2018 Published online: 18 May 2018

\footnotetext{
References

1. Medlock JM, Hansford KM, Bormane A, Derdakova M, Estrada-Peña A George JC, et al. Driving forces for changes in geographical distribution of Ixodes ricinus in Europe. Parasit Vectors. 2013;6:1.

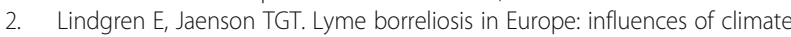
and climate change, epidemiology, ecology and adaptation measures. Report no: EUR/04/5046250. Copenhagen: World Health Organization; 2006.
} 
3. Wilking $\mathrm{H}$, Stark $\mathrm{K}$. Trends in surveillance data of human Lyme borreliosis from six federal states in eastern Germany, 2009-2012. Ticks Tick Borne Dis. 2014;5:219-24.

4. Lohr B, Müller I, Mai M, Norris DE, Schöffski O, Hunfeld KP. Epidemiology and cost of hospital care for Lyme borreliosis in Germany: lessons from a health care utilization database analysis. Ticks Tick Borne Dis. 2015;6:56-62.

5. Waindok P, Schicht S, Fingerle V, Strube C. Lyme borreliae prevalence and genospecies distribution in ticks removed from humans. Ticks Tick Borne Dis. 2017:8:709-14

6. Siński E, Welc-Falęciak R, Zajkowska J. Borrelia miyamotoi: a human tickborne relapsing fever spirochete in Europe and its potential impact on public health. Adv Med Sci. 2016:61:255-60.

7. Crowder CD, Carolan HE, Rounds MA, Honig V, Mothes B, Haag H, et al. Prevalence of Borrelia miyamotoi in IXodes ticks in Europe and the United States. Emerg Infect Dis. 2014;20:1678-82.

8. Fukunaga M, Takahashi Y, Tsuruta Y, Matsushita O, Ralph D, McClelland M, et al. Genetic and phenotypic analysis of Borrelia miyamotoi sp. nov., isolated from the ixodid tick Ixodes persulcatus, the vector for Lyme disease in Japan. Int J Syst Bacteriol. 1995;45:804-10.

9. Tappe J, Jordan D, Janecek E, Fingerle V, Strube C. Revisited: Borrelia burgdorferi sensu lato infections in hard ticks (Ixodes ricinus) in the city of Hanover (Germany). Parasit Vectors. 2014;7:441.

10. Tappe J, Jordan D, Janecek E, Fingerle V, Strube C. Erratum to: Revisited: Borrelia burgdorferi sensu lato infections in hard ticks (Ixodes ricinus) in the city of Hanover (Germany). Parasit Vectors. 2016;9:249.

11. Strube C, Montenegro VM, Epe C, Eckelt E, Schnieder T. Establishment of a minor groove binder-probe based quantitative real time PCR to detect Borrelia burgdorferi sensu lato and differentiation of Borrelia spielmanii by osp A-specific conventional PCR. Parasit Vectors. 2010;3:69.

12. May K, Jordan D, Fingerle V, Strube C. Borrelia burgdorferi sensu lato and coinfections with Anaplasma phagocytophilum and Rickettsia spp. in Ixodes ricinus in Hamburg, Germany. Med Vet Entomol. 2015;29:425-9.

13. Franke J, Hildebrandt A, Meier F, Straube E, Dorn W. Prevalence of Lyme disease agents and several emerging pathogens in questing ticks from the German Baltic coast. J Med Entomol. 2011:48:441-4.

14. Fingerle V, Munderloh UG, Liegl G, Wilske B. Coexistence of ehrlichiae of the phagocytophila group with Borrelia burgdorferi in Ixodes ricinus from southern Germany. Med Microbiol Immunol. 1999;188:145-9.

15. Bingsohn L, Beckert A, Zehner R, Kuch U, Oehme R, Kraiczy $P$, et al. Prevalences of tick-borne encephalitis virus and Borrelia burgdorferi sensu lato in Ixodes ricinus populations of the Rhine-Main region, Germany. Ticks Tick Borne Dis. 2013:4:207-13.

16. Schicht S, Junge S, Schnieder T, Strube C. Prevalence of Anaplasma phagocytophilum and coinfection with Borrelia burgdorferi sensu lato in the hard tick Ixodes ricinus in the city of Hanover (Germany). Vector Borne Zoonotic Dis. 2011;11:1595-7.

17. Schicht S, Schnieder T, Strube C. Rickettsia spp. and coinfections with other pathogenic microorganisms in hard ticks from northern Germany. J Med Entomol. 2012;49:766-71.

18. Eshoo MW, Crowder CD, Carolan HE, Rounds MA, Ecker DJ, Haag H, et al. Broad-range survey of tick-borne pathogens in southern Germany reveals a high prevalence of Babesia microti and a diversity of other tick-borne pathogens. Vector Borne Zoonotic Dis. 2014;14:584-91.

19. Venczel $R$, Knoke L, Pavlovic M, Dzaferovic E, Vaculova T, Silaghi C, et al. A novel duplex real-time PCR permits simultaneous detection and differentiation of Borrelia miyamotoi and Borrelia burgdorferi sensu lato. Infection. 2016:44:47-55.

20. Krause PJ, Fish D, Narasimhan S, Barbour AG. Borrelia miyamotoi infection in nature and in humans. Clin Microbiol Infect. 2015;21:631-9.

21. Boden $K$, Lobenstein S, Hermann B, Margos G, Fingerle V. Borrelia miyamotoi-associated neuroborreliosis in immunocompromised person. Emerg Infect Dis. 2016;22:1617-20.

22. Gugliotta JL, Goethert HK, Berardi VP, Telford SR 3rd. Meningoencephalitis from Borrelia miyamotoi in an immunocompromised patient. N Engl J Med. 2013;368:240-5.

23. Blazejak K, Janecek E, Strube C. A 10-year surveillance of Rickettsiales (Rickettsia spp. and Anaplasma phagocytophilum) in the city of Hanover, Germany, reveals Rickettsia spp. as an emerging pathogen in ticks. Parasit Vectors. 2017;10:588.

24. Estrada-Peña A, Bouattour A, Camicas JL, Walker AR. Ticks of domestic animals in the Mediterranean region: a guide to identification of species. Zaragoza, Spain: University of Zaragoza; 2004.
25. Tappe J, Strube C. Anaplasma phagocytophilum and Rickettsia spp. infections in hard ticks (Ixodes ricinus) in the city of Hanover (Germany): revisited. Ticks Tick Borne Dis. 2013;4:432-8

26. Tappe J, Strube C. Corrigendum to "Anaplasma phagocytophilum and Rickettsia spp. infections in hard ticks (Ixodes ricinus) in the city of Hanover (Germany): Revisited"[Ticks Tick Borne Dis. 4 (2013) 432-438]. Ticks Tick Borne Dis. 2016;7:1059-60

27. Alekseev AN, Dubinina HV, Van De Pol I, Schouls LM. Identification of Ehrlichia spp. and Borrelia burgdorferi in Ixodes ticks in the Baltic regions of Russia. J Clin Microbiol. 2001;39:2237-42.

28. Rijpkema SG, Molkenboer MJ, Schouls LM, Jongejan F, Schellekens JF. Simultaneous detection and genotyping of three genomic groups of Borrelia burgdorferi sensu lato in Dutch Ixodes ricinus ticks by characterization of the amplified intergenic spacer region between $5 S$ and 235 rRNA genes. J Clin Microbiol. 1995:33:3091-5.

29. Gern L, Douet V, López Z, Rais O, Cadenas FM. Diversity of Borrelia genospecies in Ixodes ricinus ticks in a Lyme borreliosis endemic area in Switzerland identified by using new probes for reverse line blotting. Ticks Tick Borne Dis. 2010;1:23-9.

30. Marie-Angele P, Lommano E, Humair PF, Douet V, Rais O, Schaad M, et al. Prevalence of Borrelia burgdorferi sensu lato in ticks collected from migratory birds in Switzerland. Appl Environ Microbiol. 2006:72:976-9.

31. Montenegro Hidalgo VM. Detection of Borrelia spp. In: Ixodes ricinus in recreation areas in Hannover (Northern Germany) PhD Thesis. Hannover, Germany: University of Veterinary Medicine. p. 2008.

32. Cochez C, Heyman P, Heylen D, Fonville M, Hengeveld P, Takken W, et al. The presence of Borrelia miyamotoi, relapsing fever spirochaete, in questing Ixodes ricinus in Belgium and in The Netherlands. Zoonoses Public Health. 2015;62:331-3.

33. Humair P, Gern L. The wild hidden face of Lyme borreliosis in Europe. Microbes Infect. 2000:2:915-22

34. Schmidt KA, Ostfeld RS. Biodiversity and the dilution effect in disease ecology. Ecology. 2001;82:609-19.

35. Casjens SR, Fraser-Liggett CM, Mongodin EF, Qiu WG, Dunn JJ, Luft BJ, et al. Whole genome sequence of an unusual Borrelia burgdorferi sensu lato isolate. J Bacteriol. 2011;193:1489-90.

36. Rudenko N, Golovchenko M, Grubhoffer L, Oliver JH Jr. Updates on Borrelia burgdorferi sensu lato complex with respect to public health Ticks Tick Borne Dis. 2011:2:123-8.

37. Kurtenbach K, Sewell HS, Ogden NH, Randolph SE, Nuttall PA. Serum complement sensitivity as a key factor in Lyme disease ecology. Infect Immun. 1998:66:1248-51.

38. Kurtenbach K, De Michelis S, Sewell HS, Etti S, Schäfer SM, Hails R, et al Distinct combinations of Borrelia burgdorferi sensu lato genospecies found in individual questing ticks from Europe. Appl Environ Microbiol. 2001;67:4926-9.

39. Gern L, Rais O. Efficient transmission of Borrelia burgdorferi between cofeeding Ixodes ricinus ticks (Acari: Ixodidae). J Med Entomol. 1996;33:189-92.

40. Hovius JW, de Wever B, Sohne M, Brouwer MC, Coumou J, Wagemakers A, et al. A case of meningoencephalitis by the relapsing fever spirochaete Borrelia miyamotoi in Europe. Lancet. 2013;382:658.

41. Richter D., Debski A., Hubalek Z, Matuschka FR., (2012) Absence of Lyme Disease Spirochetes in larval Ixodes ricinus ticks. Vector Borne Zoonotic Dis. 2012;12:21-27

42. van Duijvendijk G, Coipan C, Wagemakers A, Fonville M, Ersoz J, Oei A, et al. Larvae of Ixodes ricinus transmit Borellia afzelii and B. miyamotoi to vertebrate hosts. Parasit Vectors. 2016:9:97.

43. Chowdri HR, Gugliotta JL, Berardi VP, Goethert HK, Molloy PJ, Sterling SL, et al. Borrelia miyamotoi infection presenting as human granulocytic anaplasmosis: a case report. Ann Intern Med. 2013;159:21-7.

44. Jahfari S, Herremans T, Platonov AE, Kuiper H, Karan LS, Vasilieva O, et al. High seroprevalence of Borrelia miyamotoi antibodies in forestry workers and individuals suspected of human granulocytic anaplasmosis in the Netherlands. New Microbes New Infect. 2014:2:144-9.

45. Thomas V, Anguita J, Barthold SW, Fikrig E. Coinfection with Borrelia burgdorferi and the agent of human granulocytic ehrlichiosis alters murine immune responses, pathogen burden, and severity of Lyme arthritis. Infect Immun. 2001;69:3359-71.

46. Holden K, Hodzic E, Feng S, Freet KJ, Lefebvre RB, Barthold SW. Coinfection with Anaplasma phagocytophilum alters Borrelia burgdorferi population distribution in C3H/HeN mice. Infect Immun. 2005;73:3440-4. 